\title{
A Retrospective Review of Parent-Reported Anxiety and Emotional Functioning in Children with Developmental Challenges After Participation in the Brain Balance ${ }^{\circledR}$ Program
}

\author{
Rebecca Jackson ${ }^{1 *}$, J. Michelle Robertson ${ }^{1,2}$ \\ 'Brain Balance Achievement Centers, USA \\ ${ }^{2}$ Department of Social and Behavioral Sciences, Friends University, Wichita, KS
}

Article Info

\section{Article Notes}

Received: December 19, 2019

Accepted: January 17, 2020

\section{*Correspondence:}

Dr. Rebecca Jackson, Vice President of Program and Outcomes, Brain Balance Achievement Centers, 400 Oyster Pt Blvd, Suite 203, South San Francisco, CA 94080; Telephone No: (919) 3978206; Email: rjackson@brainbalancecenters.com.

(C) 2020 Jackson $R$. This article is distributed under the terms of the Creative Commons Attribution 4.0 International License.

\section{Keywords:}

Development

Mental health

Depression

Intervention

Behavioral therapy

Drug-free

\section{Abstract}

Children with developmental or learning challenges often have cooccurring difficulties in emotional functioning. There is a longstanding need to identify effective nonpharmacologic treatments to address these emotional difficulties. However, few studies have examined training programs that are multimodal in nature and their effects specifically on mental well-being. The purpose of this study is to estimate the impact of an integrative multimodal training program (Brain Balance) on mental well-being in children and adolescents with developmental or learning challenges, as measured by parental surveys. A retrospective review was performed on 4 years of archived survey responses from parents of enrolled students (4-17 years; $70.8 \%$ male and $29.2 \%$ female) before and after program participation. Before program participation, all students tested below age-appropriate developmental levels. The Brain Balance program consisted of three 1-hour in-center sessions per week (45 minutes of sensorimotor stimulation and 15 minutes of academic activities), along with other multimodal activities targeting proprioception and balance, vestibular functioning, fine motor skills, rhythm and timing, auditory and visual processing, and retained primitive reflexes. Parental responses showed that, after 5-6 months of program participation, $75 \%$ of children showed up to $25 \%$ improvement, and $25 \%$ of children demonstrated 60 $85.7 \%$ improvement, in the following areas: panic/anxiety attacks, worrying, depression-like symptoms, mood, obsessive thoughts or behaviors, social withdrawal, pessimism, emotional regulation, emotional self-awareness, and emotional expressiveness. These findings suggest the potential value of nonpharmacologic training programs in improving mental well-being in children and adolescents with developmental or learning challenges, especially programs that are comprehensive and multimodal.

\section{Introduction}

Children who face developmental challenges often have cooccurring difficulties in emotional functioning. A heightened risk for emotional difficulties, such as symptoms of anxiety and depression, has been reported in children with developmental issues in a range of areas, including motor, sensory, learning, and/ or attentional functioning ${ }^{1-4}$. For example, children and adolescents with poor motor skills experience higher anxiety levels and risk of psychopathology compared to peers with typically developing motor skills ${ }^{5,6}$. Similarly, children and adolescents with deficits in attention ${ }^{7-9}$ or sensory functioning ${ }^{10,11}$ often also have anxiety disorders. In fact, symptoms of sensory hyper-responsivity at the preschool age positively predict anxiety symptoms at elementary school ages ${ }^{12}$. In line with these findings, children with learning differences, such as developmental dyslexia or other literacy 
difficulties, have a high risk for social, emotional, and behavioral problems, and often show early signs of anxiety and depression ${ }^{13-15}$. These issues in emotional functioning may persist into adulthood and negatively affect mental health outcomes and adaptive behavior ${ }^{16,17}$.

It remains unclear the extent to which emotional difficulties in developmental conditions are integral features of the condition itself or just experiential consequences of a child's associated challenges. It is possible that the comorbidity of developmental challenges and emotional difficulties may stem from stressors arising from the failure to achieve age-appropriate functional skills and consequent difficulties in academic performance ${ }^{15,18}$ and/or could stem from a neurobiological vulnerability. For example, abnormalities in functional connectivity between the amygdala and medial prefrontal cortex - regions of the core neurocircuitry critical for the regulation and expression of emotional behavior - have been repeatedly reported across studies of adolescents and adults who have, or are at risk for, internalizing conditions such as anxiety and depression ${ }^{19-24}$. Various behavioral approaches, including training, practice, and behavioral therapies, have been shown to positively alter both brain connectivity and functional outcomes ${ }^{25-30}$, as well as parental reports of functional improvements in their children ${ }^{31}$. Given these findings, training programs during childhood may play a critical role in addressing challenges related to emotional functioning and anxiety in children with developmental issues, especially as there is a pressing need to identify and characterize effective nonpharmacologic treatments to target these emotional challenges.

Many conventional behavioral therapies focus on a single area of development. For example, physical therapy typically concentrates on improving gross motor skills; sensory integration therapy focuses on sensory processing issues. However, evidence from neurobiological and behavioral studies indicate a close interplay among sensory, motor, cognitive, and emotional areas of functioning in children $^{32-35}$, suggesting that the exploration of more comprehensive, integrative approaches may be warranted. The purpose of this study is to estimate the impact of an integrative whole-child approach (Brain Balance ${ }^{\circledR}$ program) on select aspects of mental health in children and adolescents with learning and developmental challenges, as measured by parental surveys. The Brain Balance program is a center-based program that aims to integrate sensory input and strengthen motor skills through regular frequency and duration of multimodal activities that target sensory functioning, motor skills, and exercises to address retained primitive reflexes, along with academic engagement, nutritional support, and complementary home-based exercises. The survey items asked parents to rate statements about aspects of their children's mental health on a numeric scale of 0 to 10 ( $0=$ not observed/ does not apply; 10 = frequently observed) before and after program participation. Although mental health comprises many different elements ${ }^{36-43}$ — including the ability to (1) learn, (2) feel, express, and manage emotions, (3) form and maintain relationships, and (4) cope with and manage change and uncertainty - the surveys used in this study focused on two specific facets of mental health related to emotional functioning and regulation (\#2 above) and anxiety (\#4 above), which will collectively be referred to in this paper as mental well-being. This paper presents a retrospective review of archived survey data collected over 4 years from parents to assess their children's mental wellbeing before and after participation in the Brain Balance program.

\section{Methods}

\section{Data source}

This review was performed retrospectively on archived survey data from parents of students enrolled at Brain Balance Achievement Center locations across the United States over a 4-year period (2015-2018). A total of 25,206 students ranging in age from 4 to 17 years $(70.8 \%$ male, $29.2 \%$ female) were enrolled in the Brain Balance program over this 4-year period, with $10 \%$ of those students choosing to not complete the program. The retrospective review included existing survey data from parents of any enrolled students who had met the criteria described in the Inclusion Criteria section below.

\section{Ethics approval}

Informed parental consent was obtained for all children prior to enrollment. The informed consent documents, study protocol, and survey items were reviewed by an institutional review board (IRB) at Advarra (Columbia, Maryland, USA), an independent organization accredited by the U.S. Office for Human Research Protections and the Association for the Accreditation of Human Research Protection Programs. The Advarra IRB determined that this retrospective data review met the requirements for exemption from IRB oversight, according to the Department of Health and Human Services regulations found at 45 CFR 46.104(d)(4).

\section{Inclusion criteria}

Prior to enrolling in the Brain Balance program, prospective students were assessed at Brain Balance centers by trained technicians who had completed a progression of training in the centers' protocols. These technicians were required to pass all station certifications and be approved while being shadowed by a trainer. Students who were eligible for enrollment in the Brain Balance program did not have any known genetic disorders 
and needed to demonstrate a developmental readiness for the program. Readiness was defined as the ability to engage with instructors and follow a one-step direction, to attempt the tasks requested, and to continue to work throughout the duration of the assessment. Re-direction and repetition of instructions both visually and verbally were allowed in our definition of readiness. The students must also have tested below age-appropriate levels, as assessed by widely used functional tests measuring a student's abilities in the following categories: fine motor skills as assessed by the Purdue Peg Board ${ }^{44}$; body coordination, timing, and strength as assessed by the Presidential Fitness Test ${ }^{45}$; interaural asymmetry as assessed by the dichotic listening test ${ }^{46}$; and visual reading fluency as assessed by the Visagraph Reading Plus ${ }^{\circledR}$ tool $^{47}$; as well as proprioception, balance, and vestibular function; auditory and visual processing; and eye coordination and movements. Enrollment criteria also required parental responses of $\geq 4$ on each survey item (see "Parental Surveys" section). This was based on criteria previously used in pain clinical trials ${ }^{48}$, where entry criteria in the study is enough pain (often a self-reported pain rating of $\geq 4$ out of 10 ) to warrant treatment. Enrolled children then participated in the Brain Balance program for a duration of 5-6 months (1 hour/day, 3 days/week), as described in more detail below.

\section{Training program}

The Brain Balance program consisted of three in-center sessions per week, with each session lasting 1 hour (45 minutes of sensorimotor stimulation and 15 minutes of academic activities), along with other multimodal activities targeting the areas described in the list below. All students went through the same series of stations. Each exercise and activity was progressive in nature and changed in duration, quantity, and complexity as the students' functional abilities improved over the course of the program. The stations consisted of the following key pieces:

- Passive sensory stimulation in the form of tactile, olfactory, visual, and auditory stimulation;

- Exercises targeting primitive and postural reflexes, which were assigned based on indicators of a retained reflex at the time of the initial assessment. The following reflexes were assessed: Moro reflex, spinal Galant reflex, rooting reflex, palmar grasp reflex, asymmetrical tonic neck reflex, symmetrical tonic neck reflex, tonic labyrinthine reflex, and Landau reflex;

- Core muscle exercises;

- Proprioceptive and balance training;

- Vestibular exercises, including rotational, translational, and anterior-to-posterior movements;

- Fine motor activities, including the palmar grasp reflex to increase muscle strength and the Purdue Peg Board to improve dexterity and speed;

- Rhythm and timing exercises, including whole-body coordination activities and use of the Interactive Metronome $^{\circledR}$, a training tool that combines the concept of a musical metronome with a computerized program that measures and improves rhythm and timing ${ }^{49}$;

- Activities that aim to enhance auditory and visual processing, as well as coordination and endurance of eye movements. More specifically, auditory engagement consisted of exposure to varying levels of auditory stimulation and activities targeting the ability to filter and rapidly process auditory information. Visual stimulation was achieved through exposure to color and light stimulation, as well as exercises that require eye coordination, timing, and speed of processing perceived information.

The academic component of the 1-hour session was based on the initial functional assessment and focused on introducing literacy and listening activities in order to increase comprehension, processing, retention, engagement, organization, and confidence.

In addition to the abovementioned in-center sessions, parents were asked to assist their children in completing daily home exercises and were also given nutritional guidance and support throughout the duration of the program. The home exercises consisted of 0-8 primitive reflexes, physical fitness activities (push-ups and sit-ups), and eye strengthening exercises. To ensure consistency in parental implemental of the at-home portions of the program, parents received in-center training on how to do the home exercises and were provided access to an online parent portal that included videos on each of the exercises as well as written instructions with photos.

\section{Parental surveys}

Surveys were administered to parents of all students prior to the initial assessment as well as part of the postprogram assessment. The purpose of the survey was to gauge parents' perspectives on their children's functioning prior to program initiation and after program completion. The survey, designed by a team of Brain Balance professionals using standard survey design method $\mathrm{s}^{50}$, consisted of 200 questions to assess parental perceptions of their children's baseline skills, attention, emotional functioning, behavior, and socialization. In the present paper, a retrospective analysis is shown for a subset of these survey items (11 items) that were focused specifically on aspects of mental well-being related to anxiety and emotional functioning and regulation. The survey items were administered in a computer-based format and used numeric rating scales based on those previously used to evaluate the effects of 
behavioral interventions and pain clinical trials ${ }^{48,51}$. Parents were instructed to rate each statement in the survey on a numeric scale of 0 to 10 ( $0=$ not observed/does not apply; 10 = frequently observed).

\section{Data analysis}

A retrospective analysis was performed on archived survey responses that had been collected from parents for four individual groups of students each year from 2015 to 2018. Little variability was observed in the data from one year to the next; therefore, the data shown are combined from all four years. For each survey item, changes were calculated in parent-reported symptom intensity as: (1) the raw symptom intensity difference [SID = SI-Post SI-Initial], and (2) the percentage symptom intensity difference $[\%$ SID $=($ SID $/$ SI-Initial $) * 100]$, where SI-Initial represents symptom intensity in the initial pre-program assessment and SI-Post represents symptom intensity in the post-program assessment.

\section{Results}

\section{Survey item "child may experience panic/anxiety attacks"}

The number of parents who provided responses to the survey item "child may experience panic/anxiety attacks" was 495 over 4 years (2015-2018). Shown in Figure 1 are data on the percentage improvement in children's panic or anxiety attacks from initial program enrollment to program completion at 5-6 months, as reported by parents. An analysis of the survey responses indicated that $75 \%$ of children showed a $20 \%$ improvement or more in panic or anxiety attacks after program participation, and $25 \%$ of children demonstrated an $85.7 \%$ improvement or more, with an average of $49.4 \%(\mathrm{SD}=45.6)$ and a median of $60 \%$. In addition, the average percentage improvement for this survey item was further broken down by participants'

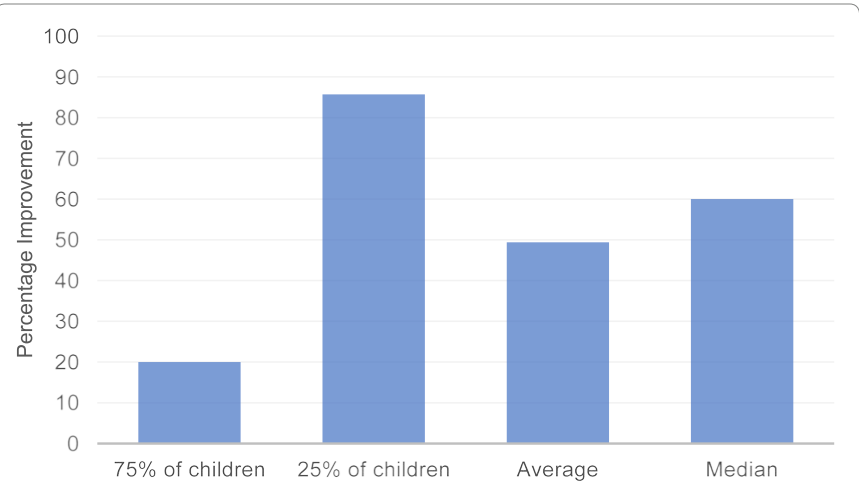

Figure 1: Effect of the Brain Balance program on parent-reported panic/anxiety attacks in children. Data are related to the parental survey item "child may experience panic/anxiety attacks." Shown are data on the percentage improvement in parental responses to this survey item from initial program enrollment to program completion at 5-6 months, along with average and median scores.

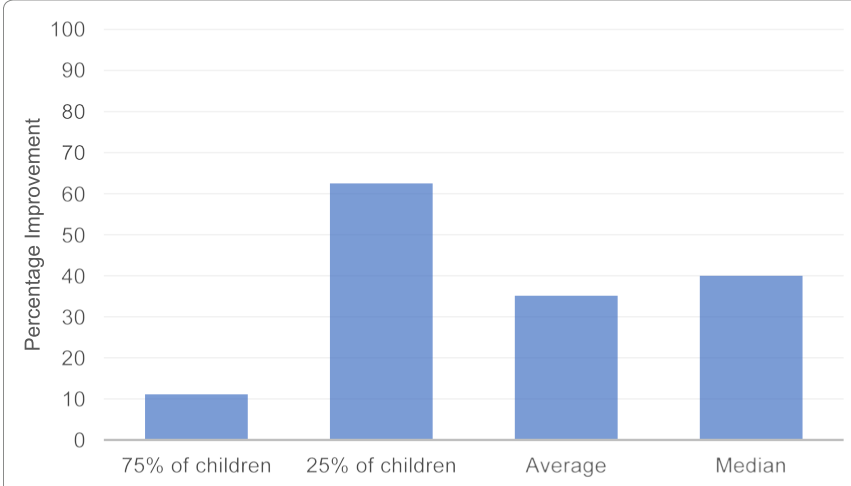

Figure 2: Effect of the Brain Balance program on parent-reported worrying in children. Data are related to the parental survey item "child worries a lot." Shown are data on the percentage improvement in parental responses to this survey item from initial program enrollment to program completion at 5-6 months, along with average and median scores.

gender ( $49 \%$ for boys, $47.5 \%$ for girls), as well as by age group (54\% for $4-6$ years old, $48 \%$ for $7-9$ years old, $49 \%$ for $10-12$ years old, $45 \%$ for $13-15$ years old, and $43 \%$ for 15-17 years old).

\section{Survey item "child worries a lot"}

The number of parents who provided responses to the survey item "child worries a lot" was 1110 over 4 years (2015-2018). Figure 2 shows data on the percentage improvement in children's worrying behavior from initial program enrollment to program completion at 5-6 months, as reported by parents. An analysis of the survey responses demonstrated that $75 \%$ of children displayed an $11.1 \%$ improvement or more in worrying behavior after program participation, and $25 \%$ of children showed a $62.5 \%$ improvement or more, with an average of $35.1 \%$ ( $\mathrm{SD}=38.7$ ) and a median of $40 \%$. Additionally, the average percentage improvement for this survey item was further broken down by participants' gender (35.8\% for boys, $33.8 \%$ for girls), as well as by age group (36\% for 4-6 years old, $35 \%$ for $7-9$ years old, $36 \%$ for $10-12$ years old, $30 \%$ for $13-15$ years old, and $31 \%$ for $15-17$ years old).

\section{Survey item "child seems depressed"}

The number of parents who provided responses to the survey item "child seems depressed" was 428 over 4 years (2015-2018). In Figure 3, data are shown on the percentage improvement in children's depression-like symptoms from initial program enrollment to program completion at 5-6 months, as reported by parents. An analysis of the survey responses demonstrated that $75 \%$ of children displayed a $25 \%$ improvement or more in depression-like symptoms after program participation, and $25 \%$ of children showed an $85.7 \%$ improvement or more, with an average of $53.1 \%$ ( $\mathrm{SD}=40.8$ ) and a median of $60 \%$. In addition, the average percentage improvement for this survey item was further 


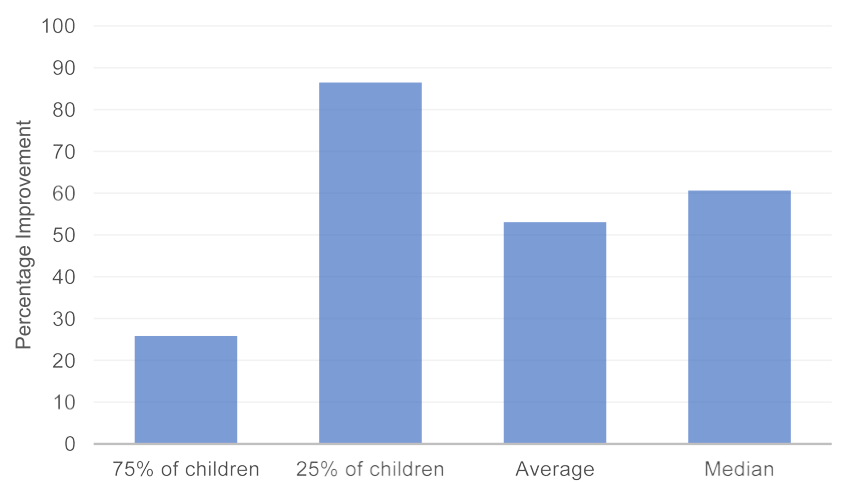

Figure 3: Effect of the Brain Balance Program on parent-reported depression-like symptoms in children. Data are related to the parental survey item "child seems depressed." Shown are data on the percentage improvement in parental responses to this survey item from initial program enrollment to program completion at 5-6 months, along with average and median scores.

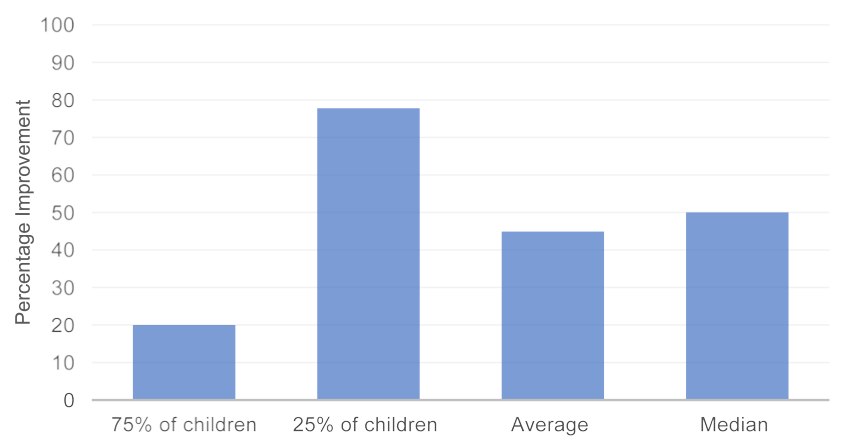

Figure 4: Effect of the Brain Balance program on parent-reported mood in children. Data are related to the parental survey item "child often appears to be unhappy." Shown are data on the percentage improvement in parental responses to this survey item from initial program enrollment to program completion at 5-6 months, along with average and median scores.

broken down by participants' gender (54.5\% for boys, $50.8 \%$ for girls), as well as by age group (74\% for 4-6 years old, $58 \%$ for $7-9$ years old, $55 \%$ for $10-12$ years old, $47 \%$ for 13-15 years old, and 39\% for 15-17 years old).

\section{Survey item "child often appears to be unhappy"}

The number of parents who provided responses to the survey item "child often appears to be unhappy" was 630 over 4 years (2015-2018). Figure 4 shows data on the percentage improvement in children's mood from initial program enrollment to program completion at 5-6 months, as reported by parents. Analysis of the survey responses indicated that $75 \%$ of children showed a $20 \%$ improvement or more in mood following program participation, and $25 \%$ of children demonstrated a $77.8 \%$ improvement or more, with an average of $44.9 \%$ (SD $=39.8$ ) and a median of $50 \%$. In addition, the average percentage improvement for this survey item was further broken down by participants' gender (47.7\% for boys, $36.5 \%$ for girls), as well as by age

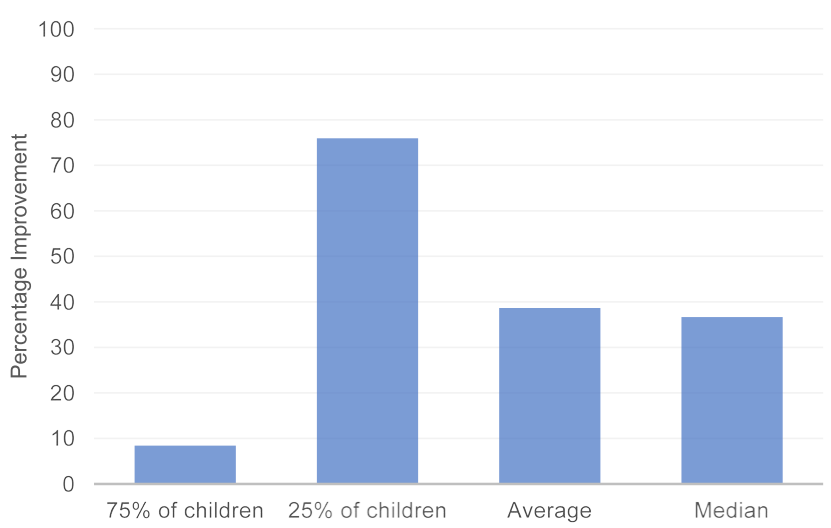

Figure 5: Effect of the Brain Balance program on parent-reported obsessive thoughts or behaviors in children. Data are related to the parental survey item "child tends to have obsessive thoughts or behaviors." Shown are data on the percentage improvement in parental responses to this survey item from initial program enrollment to program completion at 5-6 months, along with average and median scores.

group ( $49 \%$ for $4-6$ years old, $47 \%$ for $7-9$ years old, $46 \%$ for $10-12$ years old, $36 \%$ for $13-15$ years old, and $36 \%$ for 15-17 years old).

\section{Survey item "child tends to have obsessive thoughts or behaviors"}

The number of parents who provided responses to the survey item "child tends to have obsessive thoughts or behaviors" was 889 over 4 years (2015-2018). Shown in Figure 5 are data on the percentage improvement in children's obsessive thoughts or behaviors from initial program enrollment to program completion at 5-6 months, as reported by parents. An analysis of the survey responses indicated that $75 \%$ of children showed an $8.4 \%$ improvement or more in obsessive thoughts or behaviors following program participation, and $25 \%$ of children demonstrated a $75.9 \%$ improvement or more, with an average of $38.6 \%$ (SD $=43.9$ ) and a median of $36.7 \%$. Additionally, the average percentage improvement for this survey item was further broken down by participants' gender (38.5\% for boys, $41.2 \%$ for girls), as well as by age group (35\% for $4-6$ years old, $41 \%$ for $7-9$ years old, $33 \%$ for $10-12$ years old, $37 \%$ for $13-15$ years old, and $40 \%$ for 15-17 years old).

\section{Survey item "child withdraws socially"}

The number of parents who provided responses to the survey item "child withdraws socially" was 689 over 4 years (2015-2018).In Figure 6, we present data on the percentage improvement in children's social withdrawal behavior from initial program enrollment to program completion at 5-6 months, as reported by parents. An analysis of the survey responses showed that $75 \%$ of children displayed a $14.3 \%$ improvement or more in social withdrawal after 


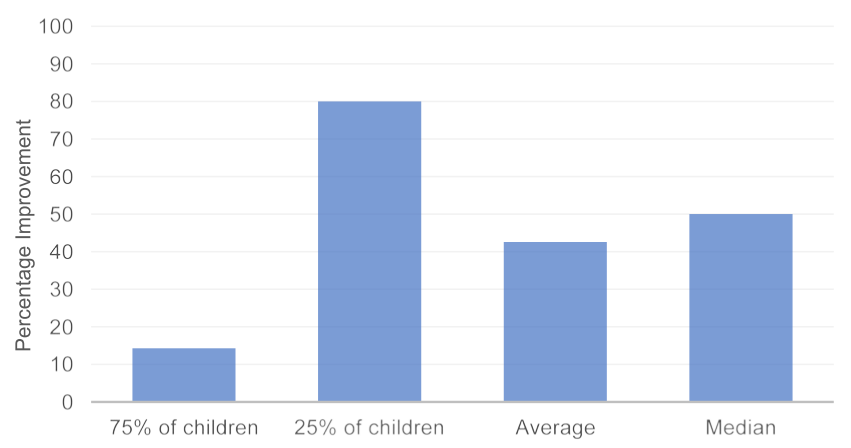

Figure 6: Effect of the Brain Balance program on parent-reported social withdrawal in children. Data are related to the parental survey item "child withdraws socially." Shown are data on the percentage improvement in parental responses to this survey item from initial program enrollment to program completion at 5-6 months, along with average and median scores.

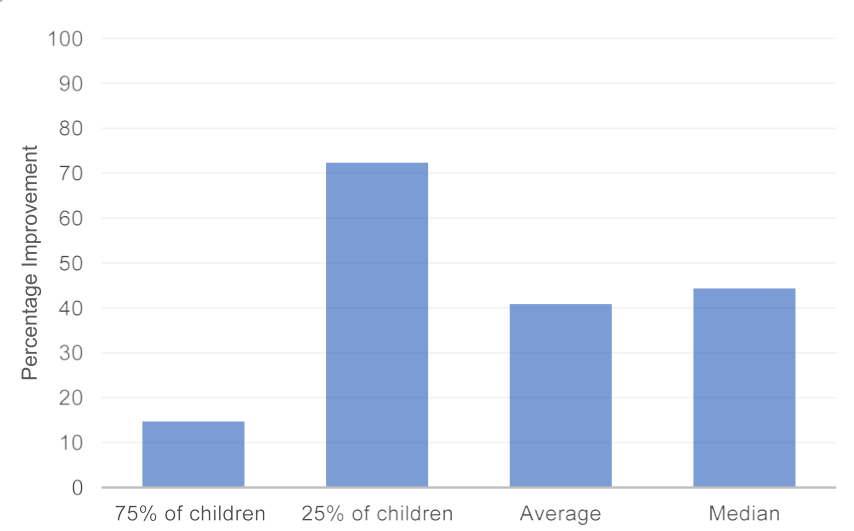

Figure 7: Effect of the Brain Balance program on parent-reported pessimism in children. Data are related to the parental survey item "child is pessimistic or extremely negative." Shown are data on the percentage improvement in parental responses to this survey item from initial program enrollment to program completion at 5-6 months, along with average and median scores.

program participation, and $25 \%$ of children demonstrated an $80 \%$ improvement or more, with an average of $42.4 \%$ ( $S D=45.3$ ) and a median of $50 \%$. In addition, the average percentage improvement for this survey item was further broken down by participants' gender $(43.5 \%$ for boys, $39 \%$ for girls), as well as by age group (49\% for 4-6 years old, $47 \%$ for $7-9$ years old, $44 \%$ for $10-12$ years old, $40 \%$ for 13-15 years old, and $29 \%$ for $15-17$ years old).

\section{Survey item "child is pessimistic or extremely negative"}

The number of parents who provided responses to the survey item "child is pessimistic or extremely negative" was 711 over 4 years (2015-2018). Figure 7 shows data on the percentage improvement in pessimism from initial program enrollment to program completion at 5-6 months, as reported by parents. An analysis of the survey responses indicated that $75 \%$ of children showed a

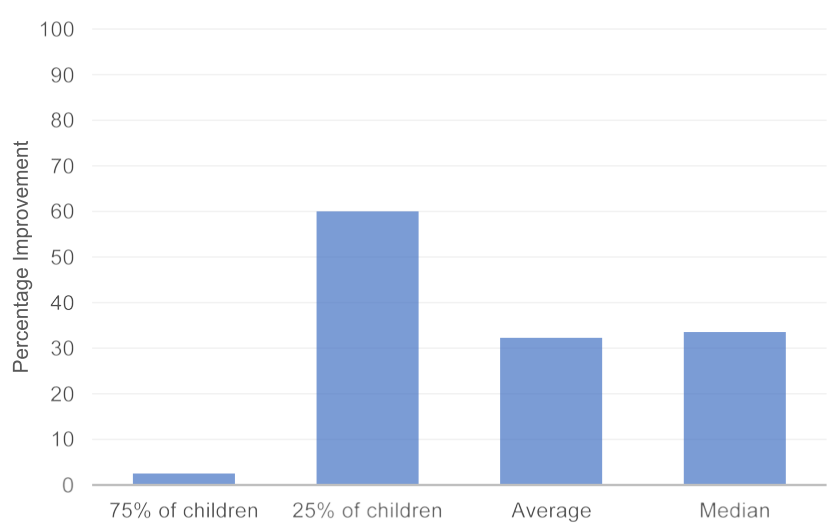

Figure 8: Effect of the Brain Balance program on parent-reported emotional regulation in children. Data are related to the parental survey item "child has difficulty suppressing or controlling emotions." Shown are data on the percentage improvement in parental responses to this survey item from initial program enrollment to program completion at 5-6 months, along with average and median scores.

$14.3 \%$ improvement or more in pessimism after program participation, and $25 \%$ of children demonstrated a $73.2 \%$ improvement or more, with an average of $41.1 \%$ (SD = 39.9 ) and a median of $42.9 \%$. Additionally, the average percentage improvement for this survey item was further broken down by participants' gender $(41.2 \%$ for boys, $39.2 \%$ for girls), as well as by age group (45\% for 4-6 years old, $45 \%$ for $7-9$ years old, $36 \%$ for $10-12$ years old, $41 \%$ for $13-15$ years old, and $41 \%$ for $15-17$ years old).

\section{Survey item "child has difficulty suppressing or controlling emotions"}

The number of parents who provided responses to the survey item "child has difficulty suppressing or controlling emotions" was 1,261 over 4 years (2015-2018). Shown in Figure 8 are data on the percentage improvement in emotional regulation from initial program enrollment to program completion at 5-6 months, as reported by parents. An analysis of the survey responses showed that $75 \%$ of children displayed a $2.5 \%$ improvement in emotional regulation following program participation, and $25 \%$ of children demonstrated a $60 \%$ improvement or more, with an average of $32.5 \%$ (SD $=40$ ) and a median of $33.3 \%$. In addition, the average percentage improvement for this survey item was further broken down by participants' gender (33.7\% for boys, $28.5 \%$ for girls), as well as by age group (29\% for $4-6$ years old, $37 \%$ for $7-9$ years old, $31 \%$ for $10-12$ years old, $29 \%$ for $13-15$ years old, and $28 \%$ for 15-17 years old).

\section{Survey item "child does not seem to be in touch with his/her feelings"}

The number of parents who provided responses to the survey item "child does not seem to be in touch with his/ 


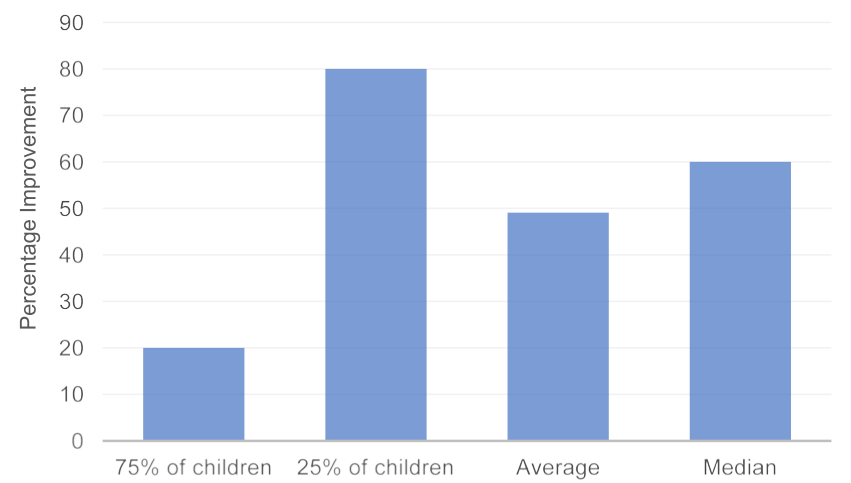

Figure 9: Effect of the Brain Balance program on parent-reported emotional self-awareness in children. Data are related to the parental survey item "child does not seem to be in touch with his/ her feelings." Shown are data on the percentage improvement in parental responses to this survey item from initial program enrollment to program completion at 5-6 months, along with average and median scores.

her feelings" was 547 over 4 years (2015-2018). In Figure 9, we present data on the percentage improvement in emotional self-awareness from initial program enrollment to program completion at 5-6 months, as reported by parents. Analysis of the survey responses indicated that $75 \%$ of children showed a $20 \%$ improvement or more in emotional self-awareness after program participation, and $25 \%$ of children demonstrated an $80 \%$ improvement or more, with an average of $49.1 \%$ ( $\mathrm{SD}=43.8$ ) and a median of $60 \%$. Additionally, the average percentage improvement for this survey item was further broken down by participants' gender (49.2\% for boys, $49 \%$ for girls), as well as by age group (58\% for 4-6 years old, $54 \%$ for 7-9 years old, $49 \%$ for $10-12$ years old, $40 \%$ for $13-15$ years old, and $38 \%$ for $15-17$ years old).

\section{Survey item "child does not demonstrate a lot of emotion"}

The number of parents who provided responses to the survey item "child does not demonstrate a lot of emotion" was 522 over 4 years (2015-2018). Figure 10a shows data on the percentage improvement in emotional expressiveness from initial program enrollment to program completion at 5-6 months, as reported by parents. An analysis of the survey responses indicated that $75 \%$ of children showed a $12.5 \%$ improvement or more in emotional expressiveness after program participation, and $25 \%$ of children demonstrated an $85.7 \%$ improvement or more, with an average of $44.4 \%(S D=46.2)$ and a median of $50 \%$. Additionally, the average percentage improvement for this survey item was further broken down by participants' gender ( $41.2 \%$ for boys, $49 \%$ for girls), as well as by age group (63\% for $4-6$ years old, $49 \%$ for $7-9$ years old, $42 \%$ for $10-12$ years old, $38 \%$ for $13-15$ years old, and $28 \%$ for 15-17 years old).
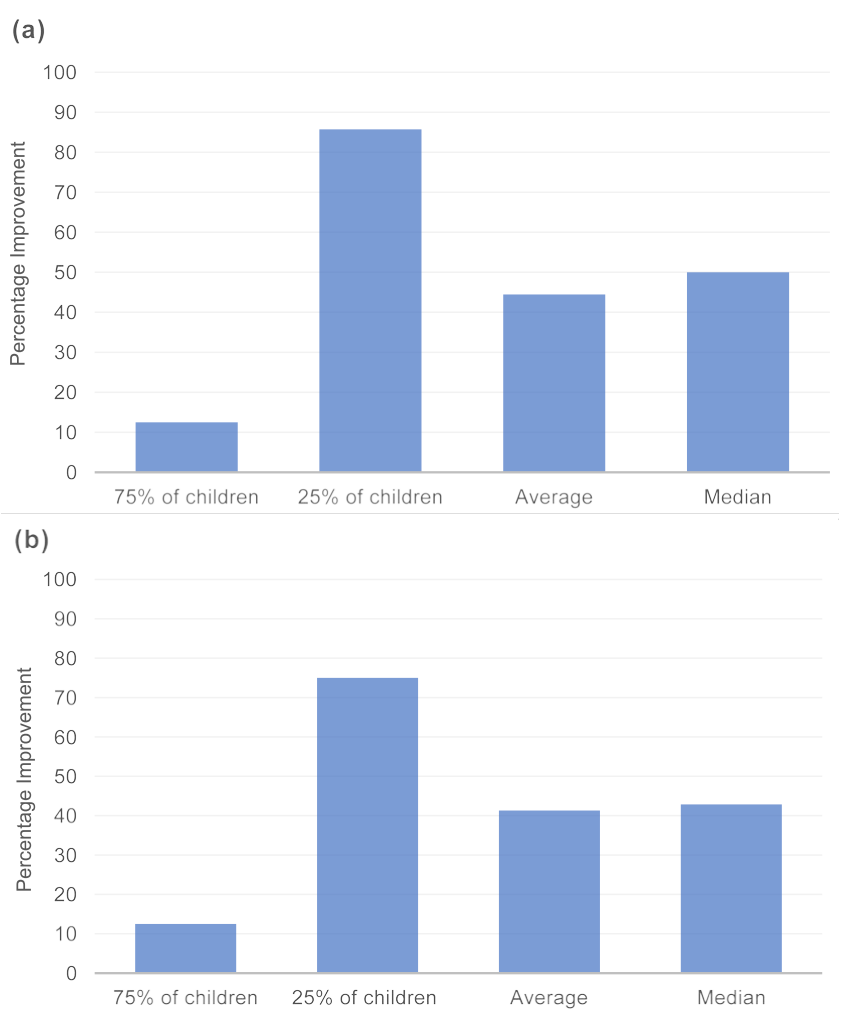

Figure 10: Effect of the Brain Balance program on parent-reported emotional expressiveness in children. Data are related to the parental survey items (a) "child does not demonstrate a lot of emotion" and (b) "child has difficulty expressing emotion." The number of parents that provided responses was 522 for the survey item in (a) and 774 for the survey item in (b) over 4 years (2015-2018). Shown are data on the percentage improvement in parental responses to these survey items from initial program enrollment to program completion at 5-6 months, along with average and median scores.

\section{Survey item "child has difficulty expressing emotion"}

The number of parents who provided responses to the survey item "child has difficulty expressing emotion" was 774 over 4 years (2015-2018). In Figure 10b, we present data on the percentage improvement in difficulties expressing emotion from initial program enrollment to program completion at 5-6 months, as reported by parents. An analysis of the survey responses indicated that, following program participation, $75 \%$ of children showed a $12.5 \%$ improvement or more in difficulties expressing emotion, and $25 \%$ of children demonstrated a $75 \%$ improvement or more, with an average of $41.3 \%$ (SD $=43.9$ ) and a median of $42.9 \%$. Note that the parental responses on survey items presented in Figures 10a and 10b, both related to the expression of emotion, are consistent with respect to percentage improvement for the majority of children. In addition, the average percentage improvement for this survey item was further broken down by participants' gender $(41.2 \%$ for boys, $39 \%$ for girls), as well as by age group (48\% for $4-6$ years old, $50 \%$ for $7-9$ years old, $37 \%$ for $10-12$ years old, $33 \%$ for $13-15$ years old, and $32 \%$ for $15-17$ years old). 


\section{Discussion}

The aim of the current study was to estimate whether participation in the Brain Balanceprogrammaybeassociated with improvements in mental well-being in children and adolescents, as measured retrospectively by a review of archived parental survey data. Many of the individual components included in this multicomponent program had been examined in previous childhood intervention studies demonstrating functional improvements after training selectively in, for example, balance and coordination ${ }^{52,53}$, sensory functioning ${ }^{54-56}$, or rhythm and timing ${ }^{49}$, as well as showing the negative impact of retained primitive reflexes on later development ${ }^{57-59}$. However, few studies had examined multimodal training that integrates many of these areas into one comprehensive program and its effects on mental well-being. In the present analysis, responses from parental surveys showed that the majority of parents reported improvements in the mental well-being of their children after 5-6 months of consistent participation in the Brain Balance program. Specifically, improvements were reported in all of the following ten areas assessed by the survey: panic/anxiety attacks, worrying, depressionlike symptoms, mood, obsessive thoughts or behaviors, social withdrawal, pessimism, emotional regulation, emotional self-awareness, and emotional expressiveness. These survey data suggest that regular participation in the Brain Balance Program may improve anxiety and emotional functioning in children and adolescents with developmental or learning challenges.

Sensorimotor activities were a substantial portion of each in-center session within the Brain Balance program. Deficits in sensorimotor functioning are a core feature across various developmental conditions $\mathrm{s}^{60-62}$ and have been linked to emotional problems ${ }^{35}$, attentional difficulties ${ }^{54,63}$, lower cognitive abilities ${ }^{32,64}$, poorer academic performance ${ }^{32,65}$, and decreased brain functional connectivity ${ }^{66,67}$. The findings of these studies are reflective of the interrelated nature of sensorimotor, cognitive, and emotional functioning during development $t^{32-35,68}$ and potentially explain the present findings of daily sensorimotor training and associated improvements in emotional functioning. In addition to the in-center sessions focused on sensory functioning and motor skills, the program also included academic activities, nutritional support, and exercises to address retained primitive reflexes. The multimodal, integrative nature of the Brain Balance program allowed for training, and therefore improvement, in multiple interrelated developmental areas, so that improvement in one area (such as sensorimotor skills) may have, in turn, positively impacted functioning in another area (such as emotional functioning).

Although there is a paucity of published data on the effects of multimodal training programs on mental well- being, there are some previous studies that explored the effects of such programs on other areas such as sensorimotor and social functioning. For example, a recent study examined the long-term effects of multimodal sensorimotor therapy consisting of vestibular, tactile, and auditory stimulation, complementary play exercises, stereotypical fetal- and infant movements, and sportsrelated gross motor exercises, in preschool- and schoolaged children ${ }^{28}$. The authors reported that, after 36 months of program participation, children with developmental coordination disorder were able to catch up with typically developing children with respect to primary reflexes, gross motor skills, and vestibular function, as shown by teacher and parent reports as well as results on sensorimotor tests. Another recent study looked at the effects of a multimodal school-based intervention in low socioeconomic areas, in which children aged 4-6 years participated for one semester in gross motor and fine motor activities, along with a social-emotional component aimed at identifying and labeling feelings, breathing, and relaxation ${ }^{29}$. This study found a significant improvement in prosocial behavior (cooperation, assertion, responsibility, and selfcontrol) in children in the intervention group compared to those in a control group, an effect that remained at an 18-month follow-up. Overall, the results of these previous studies indicate that sensorimotor issues and social skills in children can be substantially improved by multimodal intervention programs. The present study extends these findings by showing that mental well-being can also similarly be improved when children engage in programs targeting multiple developmental areas.

The improvements in mental well-being reported by parents in this survey analysis are noteworthy considering that these improvements occurred through a nonpharmacologic approach. The need to identify effective, safe, nonpharmacologic treatments for emotional challenges during development is pressing, as medication compliance is often low $^{69}$ and there are risks of adverse events in pediatric populations ${ }^{70}$. In most cases, emotional challenges such as anxiety arising during childhood and adolescence tend to persist into adulthood, requiring long-term treatment planning. However, long-term neurobiological and behavioral consequences of prolonged use of psychiatric drugs initiated during development have not yet been fully characterized. The nonpharmacologic nature of the Brain Balance program may make it more conducive to higher compliance and longer-term participation, particularly for children with moderate challenges, thereby allowing longer-term effects on mental well-being.

In this study, we chose to analyze parental surveys because parental self-reports have been demonstrated to be reliable and accurate. Studies have shown that parents' 
concerns and observations are as accurate as some highquality screening tests and that parents are equally able to raise important concerns regarding their children's development regardless of differences in education and child-rearing experience ${ }^{71,72}$. Research in progress aims to extend the current findings by analyzing parental survey responses in additional categories, including academic performance and attention. However, one limitation of the present analysis is that survey data alone obviously do not provide a full picture of the effects of Brain Balance program participation; therefore, ongoing studies are also using direct assessment measures of the children themselves, which would complement the survey responses obtained from parents.

Another limitation of the present analysis was its inclusion of a large group of enrolled students that was heterogeneous with respect to developmental and learning challenges, preventing any determination of whether the effects of program participation may have varied depending on the students' specific diagnoses, if any. Therefore, other ongoing studies are using direct assessment measures to investigate the effects of the Brain Balance program on subsets of children with specific neurodevelopmental diagnoses, such as attention-deficit hyperactivity disorder ${ }^{73}$. The present analysis of parental surveys is a first step toward estimating the potential effects of Brain Balance program participation on mental well-being in a broad population of children and adolescents who tested below age-appropriate developmental levels.

In conclusion, the present data suggest the potential value of nonpharmacologic training programs in improving anxiety and emotional functioning in children and adolescents with developmental and learning challenges, especially programs that comprehensively target multiple developmental areas, using a whole-child approach. The data point to the efficacy of this approach within the Brain Balance program, as demonstrated by parental reports of improved mental well-being after consistent program participation.

\section{Conflicts of Interest}

The primary author works for Brain Balance Achievement Centers but has no financial stake in the outcome of this study or in the publication of the results.

\section{Funding}

No specific funding was received for this study.

\section{References}

1. Cairney J, Rigoli D, Piek J. Developmental coordination disorder and internalizing problems in children: the environmental stress hypothesis elaborated. Dev Rev. 2013; 33(3): 224-238.

2. Caplan B, Neece CL, Baker BL. Developmental level and psychopathology: comparing children with developmental delays to chronological and mental age matched controls. Res Dev Disabil. 2015; 37: 143-51.

3. Harrowell I, Hollén L, Lingam R, et al. Mental health outcomes of developmental coordination disorder in late adolescence. Dev Med Child Neurol. 2017; 59(9): 973-979.

4. van den Heuvel M, Jansen DE, Reijneveld SA, et al. Identification of emotional and behavioral problems by teachers in children with developmental coordination disorder in the school community. Res Dev Disabil. 2016; 51-52: 40-48.

5. Skinner RA, Piek JP. Psychosocial implications of poor motor coordination in children and adolescents. Hum Mov Sci. 2001; 20: 73-94.

6. Green D, Baird G, Sugden D. A pilot study of psychopathology in Developmental Coordination Disorder. Child Care Health Dev. 2006; 32(6): 741-750.

7. Bowen R, Chavira DA, Bailey K, et al. Nature of anxiety comorbid with attention deficit hyperactivity disorder in children from a pediatric primary care setting. Psychiatry Res. 2008; 157(1-3): 201-209.

8. Jarrett MA, Ollendick TH. A conceptual review of the comorbidity of attention-deficit/hyperactivity disorder and anxiety: implications for future research and practice. Clin Psychol Rev. 2008; 28(7): 12661280.

9. Jensen PS, Hinshaw SP, Kraemer HC, et al. ADHD comorbidity findings from the MTA study: comparing comorbid subgroups. J Am Acad Child Adolesc Psychiatry. 2001; 40(2): 147-158.

10. Lane SJ, Reynolds S, Thacker L. Sensory over-responsivity and ADHD: differentiating using electrodermal responses, cortisol, and anxiety. Front Integr Neurosci. 2010;4:8.

11. Pfeiffer B, Kinnealey M, Reed C, et al. Sensory modulation and affective disorders in children and adolescents with Asperger's disorder. Am J Occup Ther. 2005; 59(3): 335-345.

12. Carpenter KLH, Baranek GT, Copeland WE, et al. Sensory overresponsivity: an early risk factor for anxiety and behavioral challenges in young children. J Abnorm Child Psychol. 2019; 47(6): 1075-1088.

13. Carroll JM, Maughan B, Goodman R, et al. Literacy difficulties and psychiatric disorders: evidence for comorbidity. J Child Psychol Psychiatry. 2005; 46(5): 524-532.

14. Hendren RL, Haft SL, Black JM, et al. Recognizing psychiatric comorbidity with reading disorders. Front Psychiatry. 2018; 9: 101.

15. Maughan B, Carroll J. Literacy and mental disorders. Curr Opin Psychiatry. 2006; 19(4): 350-354.

16. Sigurdsson E, Van Os J, Fombonne E. Are impaired childhood motor skills a risk factor for adolescent anxiety? Results from the 1958 U.K. birth cohort and the National Child Development Study. Am J Psychiatry. 2002; 159(6): 1044-1046.

17. van Os J, Jones P, Lewis G, et al. Developmental precursors of affective illness in a general population birth cohort. Arch Gen Psychiatry. 1997; 54(7): 625-631.

18. Haft SL, Duong PH, Ho TC, et al. Anxiety and attentional bias in children with specific learning disorders. J Abnorm Child Psychol. 2019; 47(3): 487-497.

19. Brown VM, Labar KS, Haswell CC, et al. Altered resting-state functional connectivity of basolateral and centromedial amygdala complexes in posttraumatic stress disorder. Neuropsychopharmacology. 2014; 39: 361-369.

20. Etkin A, Prater KE, Schatzberg AF, et al. Disrupted amygdalar subregion functional connectivity and evidence of a compensatory network in generalized anxiety disorder. Arch Gen Psychiatry. 2009; 66: 1361-1372. 
21. Hare TA, Tottenham N, Galvan A, et al. Biological substrates of emotional reactivity and regulation in adolescence during an emotional go-nogo task. Biol Psychiatry. 2008; 63: 927-934.

22. Johnstone T, van Reekum CM, Urry HL, et al. Failure to regulate: counterproductive recruitment of top-down prefrontal-subcortical circuitry in major depression. J Neurosci. 2007; 27: 8877-8884.

23. Marusak HA, Thomason ME, Peters C, et al. You say 'prefrontal cortex' and I say 'anterior cingulate': meta-analysis of spatial overlap in amygdala-to-prefrontal connectivity and internalizing symptomology. Transl Psychiatry. 2016; 6(11): e944.

24. Roy AK, Fudge JL, Kelly C, et al. Intrinsic functional connectivity of amygdala-based networks in adolescent generalized anxiety disorder. J Am Acad Child Adolesc Psychiatr. 2013; 52: 290-299.e2.

25. Dresler T, Bugden S, Gouet C, et al. A translational framework of educational neuroscience in learning disorders. Front Integr Neurosci. 2018; $12: 25$.

26. Horowitz-Kraus T, Toro-Serey C, DiFrancesco M. Increased restingstate functional connectivity in the cingulo-opercular cognitivecontrol network after intervention in children with reading difficulties. PLoS One. 2015; 10(7): e0133762.

27. Kelly C, Castellanos FX. Strengthening connections: functional connectivity and brain plasticity. Neuropsychol Rev. 2014; 24(1): 6376.

28. Niklasson M, Norlander T, Niklasson I, et al. Catching-up: children with developmental coordination disorder compared to healthy children before and after sensorimotor therapy. PLoS One. 2017; 12(10): e0186126.

29. Piek JP, Kane R, Rigoli D, et al. Does the Animal Fun program improve social-emotional and behavioural outcomes in children aged 4-6 years? Hum Mov Sci. 2015; 43: 155-163.

30. Posner MI, Rothbart MK, Voelker P. Developing brain networks of attention. Curr Opin Pediatr. 2016; 28(6): 720-724.

31. Grigg TM, Fox-Turnbull W, Culpan I. Retained primitive reflexes: perceptions of parents who have used Rhythmic Movement Training with their children. J Child Health Care. 2018; 22(3): 406-418.

32. Davis AS, Pass LA, Finch WH, et al. The canonical relationship between sensory-motor functioning and cognitive processing in children with attention-deficit/hyperactivity disorder. Arch Clin Neuropsychol. 2009; 24(3): 273-286.

33. Diamond A. Close interrelation of motor development and cognitive development and of the cerebellum and prefrontal cortex. Child Dev. 2000; 71(1): 44-56.

34. Hadders-Algra M. Inspiring infancy: interrelations between sensory, motor, and cognitive abilities during typical and atypical development. Dev Med Child Neurol. 2016; 58(Suppl 4): 1-2.

35. Mancini VO, Rigoli D, Roberts LD, et al. The relationship between motor skills and psychosocial factors in young children: A test of the elaborated environmental stress hypothesis. Br J Educ Psychol. 2018; 88(3): 363-379.

36. Bhugra D, Till A, Sartorius N. What is mental health? Int J Soc Psychiatry. 2013; 59(1): 3-4.

37. Davydov DM, Stewart R, Ritchie K, et al. Resilience and mental health. Clin Psychol Rev. 2010; 30: 479-495.

38. Manwell LA, Barbic SP, Roberts K, et al. What is mental health? Evidence towards a new definition from a mixed methods multidisciplinary international survey. BMJ Open. 2015; 5(6): e007079.

39. Oldehinkel AJ. Editorial: Improving children's mental health. What does that mean, actually? J Child Psychol Psychiatry. 2019; 60(8): 825-827.
40. Orpana H, Vachon J, Dykxhoorn J, et al. Monitoring positive mental health and its determinants in Canada: the development of the Positive Mental Health Surveillance Indicator Framework. Health Promot Chronic Dis Prev Can. 2016; 36(1): 1-10.

41. Westerhof GJ, Keyes CL. Mental illness and mental health: the two continua model across the lifespan. J Adult Dev. 2010; 17(2): 110-119.

42. Vaillant GE. Positive mental health: is there a cross-cultural definition? World Psychiatry. 2012; 11(2): 93-99.

43. World Health Organization. Mental health: strengthening our response. Fact Sheet No. 220. 2018. http://www.who.int/ mediacentre/factsheets/fs220/en/. Accessed September 23, 2019.

44. Squillace M, Ray S, Milazzo M. Changes in gross grasp strength and fine motor skills in adolescents with pediatric multiple sclerosis. Occup Ther Health Care. 2015; 29(1): 77-85.

45. George CL, Oriel KN, Blatt PJ, et al. Impact of a community-based exercise program on children and adolescents with disabilities. J Allied Health. 2011; 40(4): e55-60.

46. Westerhausen R, Kompus K. How to get a left-ear advantage: a technical review of assessing brain asymmetry with dichotic listening. Scand J Psychol. 2018; 59(1): 66-73.

47. Solan HA, Shelley-Tremblay J, Larson S, et al. Silent word reading fluency \& temporal vision processing differences between good and poor readers. J Behav Optom. 2006; 17(6): 149-157.

48. Farrar JT, Polomano RC, Berlin JA, et al. A comparison of change in the 0-10 numeric rating scale to a pain relief scale and global medication performance scale in a short-term clinical trial of breakthrough pain intensity. Anesthesiology. 2010; 112(6): 1464-1472.

49. Shaffer RJ, Jacokes LE, Cassily JF, et al. Effect of interactive metronome training on children with ADHD. Am J Occup Ther. 2001; 55(2): 155-162.

50. Jones TL, Baxter MA, Khanduja V. A quick guide to survey research. Ann R Coll Surg Engl. 2013; 95(1): 5-7.

51. Volpe RJ, Briesch AM, Gadow KD. The efficiency of behavior rating scales to assess inattentive-overactive and oppositional-defiant behaviors: applying generalizability theory to streamline assessment. J Sch Psychol. 2011; 49(1): 131-155.

52. Fong SS, Guo X, Liu KP, et al. Task-specific balance training improves the sensory organisation of balance control in children with developmental coordination disorder: a randomised controlled trial. Sci Rep. 2016; 6: 20945.

53. Wälchli M, Ruffieux J, Mouthon A, et al. Is young age a limiting factor when training balance? Effects of child-oriented balance training in children and adolescents. Pediatr Exerc Sci. 2018; 30(1): 176-184.

54. Padmanabha H, Singhi P, Sahu JK, et al. Home-based sensory interventions in children with autism spectrum disorder: a randomized controlled trial. Indian J Pediatr. 2019; 86(1): 18-25.

55. Hazen EP, Stornelli JL, O'Rourke JA, et al. Sensory symptoms in autism spectrum disorders. Harv Rev Psychiatry. 2014; 22(2): 112-124.

56. Woo CC, Donnelly JH, Steinberg-Epstein R, et al. Environmental enrichment as a therapy for autism: A clinical trial replication and extension. Behav Neurosci. 2015; 129(4): 412-422.

57. Gieysztor EZ, Choińska AM, Paprocka-Borowicz M. Persistence of primitive reflexes and associated motor problems in healthy preschool children. Arch Med Sci. 2018; 14(1): 167-173.

58. Konicarova J, Bob P, Raboch J. Persisting primitive reflexes in medication-naïve girls with attention-deficit and hyperactivity disorder. Neuropsychiatr Dis Treat. 2013; 9: 1457-1461.

59. McPhillips M, Sheehy N. Prevalence of persistent primary reflexes and motor problems in children with reading difficulties. Dyslexia. 2004; 10(4): 316-338. 
60. Fournier KA, Hass CJ, Naik SK, et al. Motor coordination in autism spectrum disorders: a synthesis and meta-analysis. J Autism Dev Disord. 2010; 40(10): 1227-1240.

61. Piek JP, Dyck MJ. Sensory-motor deficits in children with developmental coordination disorder, attention deficit hyperactivity disorder and autistic disorder. Hum Mov Sci. 2004; 23(3-4): 475-488.

62. Shum SB, Pang MY. Children with attention deficit hyperactivity disorder have impaired balance function: involvement of somatosensory, visual, and vestibular systems. J Pediatr. 2009; 155(2): 245-9.

63. Konicarova J, Bob P, Raboch J. Balance deficits and ADHD symptoms in medication-naïve school-aged boys. Neuropsychiatr Dis Treat. 2014; 10: 85-88.

64. Piek JP, Dawson L, Smith LM, et al. The role of early fine and gross motor development on later motor and cognitive ability. Hum Mov Sci. 2008; 27(5): 668-681.

65. Macdonald K, Milne N, Orr R, et al. Relationships between motor proficiency and academic performance in mathematics and reading in school-aged children and adolescents: a systematic review. Int J Environ Res Public Health. 2018; 15(8).

66. Kim SM, Hyun GJ, Jung TW, et al. Balance deficit and brain connectivity in children with attention-deficit/hyperactivity disorder. Psychiatry Investig. 2017; 14(4): 452-457.

67. McLeod KR, Langevin LM, Goodyear BG, et al. Functional connectivity of neural motor networks is disrupted in children with developmental coordination disorder and attention-deficit/hyperactivity disorder. Neuroimage Clin. 2014; 4: 566-575.
68. Smith LB, Sheya A. Is cognition enough to explain cognitive development? Top Cogn Sci. 2010; 2(4): 725-735.

69. Perwien A, Hall J, Swensen A, et al. Stimulant treatment patterns and compliance in children and adults with newly treated attentiondeficit/hyperactivity disorder. J Manag Care Pharm. 2004; 10: 122129.

70. Storebø OJ, Faltinsen E, Zwi M, et al. The jury is still out on the benefits and harms of methylphenidate for children and adolescents with attention-deficit/hyperactivity disorder. Clin Pharmacol Ther. 2018; 104(4): 606-609.

71. Glascoe FP, Dworkin PH. The role of parents in the detection of developmental and behavioral problems. Pediatrics. 1995; 95(6): 829-836.

72. Glascoe FP. Evidence-based approach to developmental and behavioural surveillance using parents' concerns. Child Care Health Dev. 2000; 26(2): 137-149.

73. Teicher MH. White paper: profound effects of Brain Balance exercises and interactive metronome on a subset of children with attention deficit hyperactivity disorder. Brain Balance Achievement Centers website. https://cdn2.hubspot.net/hubfs/3798961/Harvard\%20White\%20 Paper\%20BB\%20August\%202019.pdf?_hssc=206352780.1.15676 96098221\&_hstc=206352780.5b9915dedbd06fa585a044737179fc1f.1552578318119.1567522826425.1567696098221.15\&_hsf$\mathrm{p}=1588301246 \&$ hsCtaTracking $=c 9 b 93 \mathrm{c} 8 \mathrm{~b}-3 \mathrm{f} 0 \mathrm{f}-4 \mathrm{~d} 65-\mathrm{b} 99 \mathrm{e}-\mathrm{eb} 0 \mathrm{c} 36 \mathrm{f}-$ 092bc\%7Cbb08ef26-4cb5-45c2-a2b0-7ee50bc904c4. June 4, 2019. Accessed October 24, 2019. 